\title{
Immunogenetic studies of juvenile dermatomyositis
}

\section{HLA antigens in patients and their families}

\author{
J. M. Friedman ${ }^{1-3}$, L. M. Pachman ${ }^{2}$, M. L. Maryjowski ${ }^{6}$, O. Jonasson ${ }^{7}$, N. D. Battles ${ }^{2,4}$,

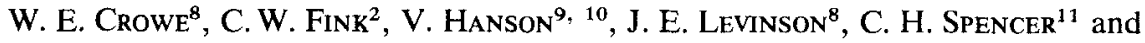 \\ D. B. SullivaN ${ }^{12}$
}

${ }^{1}$ Division of Clinical Genetics, Department of Obstetrics and Gynecology, ${ }^{2}$ Department of Pediatrics, ${ }^{3}$ Cecil H. and Ida Green Center for Reproductive Biology Sciences, University of Texas Health Science Center and the ${ }^{4}$ Scottish Rite Hospital, Dallas, Texas; ${ }^{5}$ Department of Pediatrics, Northwestern University Medical School, 'Children's Memorial Hospital, 'Department of Surgery, Abraham Lincoln School of Medicine, University of Illinois, Chicago, Illinois; ${ }^{8}$ University of Cincinnati Medical Center, Cincinnati, Ohio; 'University of Southern California Medical School, ${ }^{10}$ Los Angeles Children's Hospital, Los Angeles, California; ${ }^{11}$ Louisiana State University Medical Center, New Orleans, Louisiana; and ${ }^{12}$ University of Michigan Hospital, Ann Arbor, Michigan, U.S.A.

\begin{abstract}
Typing for HLA-A and -B antigens was performed on 87 children with definite juvenile dermatomyositis (JDMS). A significantly increased frequency of HLA-B8 (estimated relative risk $=2.8, P_{\mathrm{c}}<0.01$ ) was observed among White patients, but not among Blacks or Latin Americans with JDMS. No abnormality of HLA haplotype segregation was observed among 38 healthy siblings of the JDMS probands.
\end{abstract}

Received for publication 17 June, accepted 26 July 1982

Juvenile dermatomyositis (JDMS) is a chronic inflammatory disease of unknown etiology which is characterized by a typical rash and symmetrical proximal myopathy with onset in childhood. We have previously reported a greater than expected frequency of HLA-B8 among 16 White patients with JDMS (Pachman et al. 1977). In order to confirm this finding and to investigate its relationship to the pathogenesis of this rare disease, we undertook a multicenter collaborative investigation of immunogenetic factors in JDMS.

\section{Material and methods}

Juvenile dermatomyositis subjects. A total of 100 patients with JDMS were ascertained from the records of Pediatric Rheumatology Clinics at Children's Memorial Hospital (Chicago), University of Michigan Hospital (Ann Arbor), Los Angeles Children's Hospital, Texas Scottish Rite Hospital (Dallas), and Children's Hospital Medical Center (Cincinnati). Ninety of these patients, including 11 of our original series of 16 (Pachman et al. 1977), were considered to have definite JDMS according to the strict criteria of Bohan $\&$ Peter (1975). Each patient exhibited the 
typical rash and three or four of the following additional features: symmetrical limb-girdle muscle weakness, histological evidence of myositis on muscle biopsy, elevated serum concentration of skeletal muscle enzymes and characteristic electromyographic abnormalities. The age at onset of disease in these patients ranged from 1-21 years, with a mean of 8.0 years. There were 28 males and 62 females. Sixty-seven of the patients were White, 11 were Black, 9 were Latin Americans, 1 was Oriental and 2 were of mixed racial origin. These last three patients were excluded from the present analysis. Of the 87 patients included in this study, 23 were from Chicago, 17 from Ann Arbor, 20 from Los Angeles, 10 from Dallas, and 17 from Cincinnati.

Family studies. The families of 15 of the Chicago patients were extensively studied. Detailed family histories were obtained with particular attention being paid to immunological and putative autoimmune diseases. Blood for HLA typing was requested from all parents and siblings of the probands. A total of 30 parents, 44 full siblings, and 2 half-siblings were tested. None had any history suggestive of JDMS or polymyositis.

HLA antigen frequency controls. Normal population frequencies of the HLA-A and -B antigens among healthy Whites, Blacks and Latin Americans were obtained from the joint report of the Eighth International Workshop on Histocompatibility (Baur \& Danilovs 1980). Population groups 11 (United States Caucasians), 2 (American Negroes) and 9 (Mexicans) were used.

HLA typing and data analysis. Typing for HLA-A and HLA-B antigens on all patients and family members was performed by the standard NIH 2-stage lymphocyte microtoxicity test using NIH plates (National In- stitute of Arthritis and Infectious Diseases 1976) in the laboratory of Dr. Olga Jonasson. The study was performed over a 5-year period during which the number of available typing sera increased and splits of several antigens occurred. To permit consistent analysis, all patients positive for antigens which had been split during the study were considered to be positive for the unsplit antigen. All patients were tested for at least 10 HLA-A antigens and at least 17 HLA-B antigens.

HLA antigen frequencies were compared in patients and controls of similar ethnic origin by Fisher's exact test. Two-tailed probabilities of less than 0.05 after multiplication by 27 to correct for the number of antigens tested were considered significant (Grumet et al. 1971). Relative risks were estimated by Woolf's method (Woolf 1955) and segregation analysis was performed using the $\mathrm{X}^{2}$ test for goodness of fit.

\section{Results}

HLA typing. HLA-A and - $\mathrm{B}$ antigens were determined in 87 White, Black, or Latin American patients with definite JDMS. Table 1 compares the antigen frequencies found in these patients with the frequencies in healthy controls of similar ethnic origin. HLA-B8 occurred significantly more often among White JDMS patients than among controls. This association is not apparent among Black or Latin American patients. The estimated relative risk of JDMS among Whites with HLA-B 8 is 2.8 with a $95 \%$ confidence interval of 1.6 to 4.8 . An interesting observation is that the estimated relative risk for JDMS among Whites with HLA-B7 is only 0.2 (95\% confidence interval $=0.06$ to 0.65 ); this association is statistically significant before correction was made for the number of antigens tested $(P=0.003)$, but not after $\left(P_{\mathrm{c}}=\right.$ 0.081 ). 
Table 1.

HLA antigen frequences in patients with juvenile dermatomyositis and in healthy controls.

\begin{tabular}{|c|c|c|c|c|c|c|}
\hline \multirow[t]{2}{*}{ HLA antigens ${ }^{+}$} & \multicolumn{2}{|c|}{ White } & \multicolumn{2}{|c|}{ Black } & \multicolumn{2}{|c|}{ Latin American } \\
\hline & Patients & Controls & Patients & Controls & Patients & Controls \\
\hline & $(N=67)$ & $(\mathrm{N}=867)$ & $(\mathrm{N}=11)$ & $(N=186)$ & $(\mathrm{N}=9)$ & $(N=85)$ \\
\hline $\mathrm{A} 1$ & .33 & .253 & .09 & .065 & .11 & .00 \\
\hline $\mathrm{A} 2$ & .57 & .474 & .00 & .296 & .33 & .46 \\
\hline A3 & .22 & .269 & .00 & .177 & .33 & .06 \\
\hline A9 (w23, w24) & .18 & .184 & .18 & .274 & .33 & .27 \\
\hline $\mathrm{A} 10(\mathrm{w} 25, \mathrm{w} 26)$ & .06 & .112 & .09 & .081 & .11 & .33 \\
\hline A11 & .10 & .120 & .00 & .016 & .11 & .07 \\
\hline \multicolumn{7}{|l|}{ A19 (w29, w30, w31, } \\
\hline w32, w33) & .28 & .287 & .73 & .533 & .22 & .43 \\
\hline A28 & .07 & .100 & .27 & .118 & .44 & .06 \\
\hline Aw34 & .00 & .005 & .27 & .119 & .00 & .02 \\
\hline \multirow[t]{2}{*}{ Aw36 } & .01 & .006 & .00 & .027 & .00 & .00 \\
\hline & $(N=67)$ & $(\mathrm{N}=867)$ & $(N=11)$ & $(N=184)$ & $(\mathrm{N}=9)$ & $(\mathrm{N}=84)$ \\
\hline B5 (w51, w52) & .07 & .121 & .09 & .049 & .33 & .17 \\
\hline $\mathrm{B} 7$ & .04 & .193 & .36 & .147 & .22 & .06 \\
\hline $\mathrm{B} 8$ & .37 & $* * .175$ & .09 & .044 & .00 & .06 \\
\hline $\mathrm{B} 12(w 44, w 45)$ & .25 & .276 & .27 & .233 & .00 & .34 \\
\hline B 13 & .04 & .052 & .00 & .022 & .11 & .05 \\
\hline B14 & .06 & .092 & .00 & .076 & .00 & .05 \\
\hline B15 (w62, w63) & .18 & .117 & .09 & .021 & .00 & .18 \\
\hline Bw16 (w38, w39) & .04 & .108 & .00 & .011 & .11 & .15 \\
\hline B17 (w57, w58) & .07 & .094 & .45 & .223 & .11 & .04 \\
\hline B18 & .09 & .091 & .09 & .082 & .00 & .00 \\
\hline Bw21 (w49, w50) & .04 & .074 & .00 & .093 & .00 & .16 \\
\hline Bw22 (w54, w55, w56) & .01 & .051 & .00 & .011 & .22 & .00 \\
\hline B27 & .10 & .075 & .00 & .027 & .00 & .05 \\
\hline Bw35 & .24 & .151 & .18 & .141 & .44 & .42 \\
\hline B37 & .01 & .033 & .00 & .011 & .00 & .01 \\
\hline B40 (w47, w48, w60, w61) & .16 & .160 & .00 & .076 & .11 & .18 \\
\hline Bw41 & .04 & .032 & .00 & .016 & .00 & .01 \\
\hline
\end{tabular}

+ Splits indicated in parentheses - see Methods.

** $P_{\mathrm{e}}<0.01$.

HLA family studies. HLA typing was performed on the parents and all available normal siblings of 15 JDMS patients. Twelve of the families were informative regarding segregation of HLA haplotypes from both parents. Segregation of HLA haplotypes was normal in unaffected siblings of JDMS pa- tients (Table 2). Seventeen siblings inherited the proband's haplotype from the father; 21 siblings inherited the other haplotype $\left(\mathrm{X}^{2}=\right.$ 0.42 ). Twenty siblings inherited the proband's haplotype from the mother; $17 \mathrm{did}$ not $\left(\mathrm{X}^{2}=\right.$ 0.24 ). 
Table 2.

Distribution of HLA haplotypes in 37 normal siblings of patients with juvenile dermatomyositis.*

\begin{tabular}{lcccc}
\hline & \multicolumn{4}{c}{ Haplotypes $^{+}$} \\
\cline { 2 - 5 } & $a / c$ & $a / d$ & $b / c$ & $b / d$ \\
\hline \multirow{2}{*}{ Expected } & $25 \%$ & $25 \%$ & $25 \%$ & $25 \%$ \\
Observed & $27 \%$ & $16 \%$ & $27 \%$ & $30 \%$ \\
\hline
\end{tabular}

* One sibling who exhibited a maternal recombinant HLA-B/DR haplotype is excluded from the table.

+ The haplotype transmitted to the proband from the father is arbitrarily designated " $a$ ", and the haplotype transmitted to the proband from the mother, "c". The other paternal and maternal haplotypes are designated " $b$ " and " $d$ ", respectively. Thus, a/c siblings have both haplotypes in common with the proband, a/d siblings share just the paternal haplotype with the proband, $b / c$ siblings share just the maternal haplotype with the proband, and $b / d$ siblings differ from the proband at both haplotypes.

\section{Discussion}

Juvenile dermatomyositis is an uncommon disease. In spite of having identified patients from five large pediatric rheumatology clinics, we were able to obtain only 90 who were appropriate for this study. HLA antigen frequencies are known to differ between races, so the probands had to be divided into racial groups for analysis. Consequently, the number of patients studied in each group was relatively small, and biologically important associations between JDMS and certain HLA types may have been missed, especially among Blacks and Latin Americans.

Any demonstration of a population association between a disease and genetic markers such as HLA antigens depends critically on selection of an appropriate control group. In addition to being of the same ethnic origin as the patients, the controls ideally should be tested in the same laboratory as the patients with the same reagents at the same time. Because of the time required to amass the study group and because these patients were drawn from five different large referral areas, no ideal control groups were available to us. We therefore used as controls normal Americans of similar ethnic origin who had been typed by the same techniques during the Eighth International Histocompatibility Workshop. This approach is probably satisfactory for HLA-A and -B typing, which is well standardized.

The data presented here substantiate the association between JDMS and HLA-B8 in Whites which we previously reported in a much smaller group of patients (Pachman et al. 1977). A similar association between HLA-B8 and polymyositis in White adults has been found by some workers (Behan et al. 1978, Hirsch et al. 1981) but not others (Cumming et al. 1977). However, these data are not strictly comparable to ours because adult polymyositis and JDMS are probably different diseases (Bohan \& Peter 1975). In fact, JDMS itself is sometimes difficult to diagnose clinically and may well be etiologically heterogeneous. In order to circumvent this problem as much as possible, we insisted that the patients included in our study have definite JDMS according to the strict criteria of Bohan \& Peter (1975).

The data reported here place JDMS into company with other HLA-B 8 associated diseases including dermatitis herpetiformis, celiac disease, myasthenia gravis, Sjogren syndrome, idiopathic Addison disease, insulin-dependent diabetes mellitus, and Graves disease (Nerup et al. 1977, Friedman \& Fialkow 1978). All of these conditions are of unknown but probably complex etiology and all exhibit autoimmune manifestations. This raises the possibility that JDMS may also be an "autoimmune disease." Autoimmunity is being studied in this series of patients, and the results of these investigations will be reported in the near future. 


\section{Acknowledgments}

Supported by grants from the U.S. Public Health Service, National Institutes of Health (AM-21589 and RR-00199), and the Illinois Chapter of the Arthritis Foundation.

\section{References}

Baur, M. P. \& Danilovs, J. A. (1980) Population analysis of HLA-A, B, C, DR, and other genetic markers. In Histocompatibility Testing 1980, ed. Terasaki, P., pp. 955-960. UCLA Tissue Typing Laboratory, Los Angeles.

Behan, W. M. H., Behan, P. O. \& Dick, H. A. (1978) HLA-B8 in polymyositis. New Engl $J$ Med 298, 1260-1261.

Bohan, A. \& Peter, J. B. (1975) Polymyositis and dermatomyositis. New Engl J Med 292, 344-347, 403-407.

Cumming, W. J. K., Hudgson, P., Lattimer, D., Sussman, M. \& Wilcox, C. B. (1977) HLA and serum complement in polymyositis. Lancet $\mathbf{2}$, 978-979.

Friedman, J. M. \& Fialkow, P. J. (1978) The genetics of Graves' disease. Clin Endocrinol Metab 7, 47-65.

Grumet, F. C., Coukell, A., Bodmer, J. G., Bodmer, W. F. \& McDevitt, H. O. (1971) Histocompatibility (HL-A) antigens associated with systemic lupus erythematosus. New Engl J Med 285, 193-196.
Hirsh, T. J., Enlow, R. W., Bias, W. B. \& Arnett, F. C. (1981) HLA-D related (DR) antigens in various kinds of myositis. Hum Immunol 3, 181-186.

National Institute of Arthritis and Infectious Diseases, National Institutes of Health (1976) NIAID Manual of Tissue Typing Techniques 1976-1977. DHEW publication number NIH 76-545, Bethesda, MD.

Nerup, J., Cathelineau, C., Seignalet, J. \& Thomsen, M. (1977) HLA and endocrine diseases. In $H L A$ and Disease, eds. Dausset, J. \& Svejgaard, A., pp. 149-167. Williams and Wilkins, Baltimore.

Pachman, L. M., Jonasson, O., Cannon, R. A. \& Friedman, J. M. (1977) HLA-B8 in juvenile dermatomyositis. Lancet 2, 567--568.

Woolf, B. (1955) On estimating the relation between blood group and disease. Ann Hum Genet 19. 251-253.

Address:

J. M. Friedman, M.D., Ph.D.

Division of Clinical Genetics

Department of Obstetrics and Gynecology

University of Texas Health Science Center at Dallas 5323 Harry Hines Boulevard

Dallas, Texas, 75235 USA. 